\title{
Update to the College of American Pathologists Reporting on Thyroid Carcinomas
}

\author{
Ronald Ghossein
}

Received: 16 January 2009/Accepted: 17 February 2009/Published online: 28 February 2009

(C) Humana 2009

\begin{abstract}
Background The reporting of thyroid carcinomas follows the recommendations of the College of American Pathologists (CAP) protocols and includes papillary carcinoma, follicular carcinoma, anaplastic carcinoma and medullary carcinoma. Despite past and recent efforts, there are a number of controversial issues in the classification and diagnosis of thyroid carcinomas (TC) that, potentially impact on therapy and prognosis of patients with TC. Discussion The most updated version of the CAP thyroid cancer protocol incorporates recent changes in histologic classification as well as changes in the staging of thyroid cancers as per the updated American Joint Commission on Cancer staging manual. Among the more contentious issues in the pathology of thyroid carcinoma include the defining criteria for tumor invasiveness. While there are defined criteria for invasion, there is not universal agreement in what constitutes capsular invasion, angioinvasion and extrathyroidal invasion. Irrespective of the discrepant views on invasion, pathologists should report on the presence and extent (focal, widely) of capsular invasion, angioinvasion and extrathyroidal extension. These findings assist clinicians in their assessment of the recurrence risk and potential for metastatic disease. It is beyond the scope of this paper to detail the entire CAP protocol for thyroid carcinomas; rather, this paper addresses some of the more problematic issues confronting pathologists in their assessment and reporting of thyroid carcinomas. Conclusion The new CAP protocol for reporting of thyroid carcinomas is a step toward improving the clinical value of the histopathologic reporting of TC. Large meticulous clinico-pathologic
\end{abstract}

R. Ghossein $(\bowtie)$

Department of Pathology, Memorial Sloan-Kettering Cancer Center, 1275 York Avenue, 10065 New York, NY, USA

e-mail: ghosseir@mskcc.org and molecular studies with long term follow up are still needed in order to increase the impact of microscopic examination on the prognosis and management of TC.

Keywords Thyroid - Carcinoma - Reporting - CAP . Vascular · Capsular - Invasion · Minimally invasive . Widely invasive · Extrathyroid - Extension - Mitosis . Necrosis $\cdot$ Margins $\cdot$ Papillary microcarcinomas

\section{Introduction}

Despite advances in the last several decades in the diagnosis of thyroid cancers, there are still many problems and controversies related to the histopathology of thyroid carcinomas. These controversies impact on the prognosis and therapy of thyroid cancer patients, as well as, on the development of cutting edge research aimed at better outcome and quality of life for these patients. These contentious issues may directly impact on the pathologic reporting of these carcinomas. In the most updated version of the College of American Pathologists (CAP) protocol for the examination of specimens from patients with carcinomas of the thyroid, attempts were made to address some of these controversies. The goal was to produce a practical document providing for more clinically relevant pathology reports. The updated CAP protocol is essentially organized in a similar fashion to the previous edition with some modifications primarily but not exclusively based on the current CAP recommendations for the reporting of all cancers.

Among the controversial issues in thyroid pathology include (but are not limited to) the histopathologic interpretation of an encapsulated (well-differentiated) thyroid follicular neoplasm, specifically in determining which of these lesions does and which does not represent the 
follicular variant of papillary thyroid carcinoma $[1,2]$. Controversies also relate to the concept of the less commonly occurring poorly-differentiated thyroid carcinoma. It is beyond the scope of the CAP protocol to provide guidelines for the histopathologic interpretation of thyroid cancers. Among the standard data elements required by the CAP, this paper addresses specific issues pathologists will confront relative to criteria of malignancy (e.g., invasiveness) and extrathyroidal extension (ETE). Unfortunately, even among the authors of the updated CAP Thyroid Cancer Protocol there was not uniformity in agreement on these issues. As such, the document echos the varying views on invasiveness and ETE. Although listed as recommended rather than required elements, mitotic activity and necrosis are important in potentially determining whether a given cancer represents a poorly-differentiated thyroid carcinoma. Finally, the issue of whether the identification of thyroid papillary microcarcinomas should engender the use of the CAP Thyroid Cancer Protocol will be discussed.

The updated CAP thyroid protocol is still a work in progress and a final consensus among the responsible authors has not been reached on all of the reporting data elements, including (but not limited to): (1) whether to report all identifiable foci of thyroid papillary microcarcinoma including incidentally identified foci or whether to limit reporting to only those foci that were detectable preoperatively (by clinical examination and/or radiographic evaluation); (2) whether to limit the use of the designation of thyroid papillary microcarcinoma to adults excluding such a designation for pediatric age groups. Consequently, the recommendations herein contained reflect the views of the author of this manuscript, as well as the senior author of the CAP protocol (Bruce M. Wenig MD ${ }^{1}$ ). Modifications to the recommendations in this manuscript based on a final consensus opinion of the entire panel may yet occur.

\section{Discussion}

Criteria and Extent of Capsular and Vascular Invasion in Follicular Carcinoma and its Variants

\section{Capsular Invasion (CI)}

The diagnosis of papillary thyroid carcinoma is entirely predicated on the presence of diagnostic nuclear features. As such, the diagnosis of papillary thyroid carcinoma can be made in the presence of an encapsulated follicular

\footnotetext{
${ }^{1}$ Bruce M. Wenig, M.D is Professor of Pathology and Chairman, Department of Pathology and Laboratory Medicine, Beth Israel Medical Center and St. Luke's and Roosevelt Hospitals, New York NY.
}

neoplasm even in the absence of invasive growth. Once a given neoplasm invades its capsule and/or shows evidence of angioinvasion (intracapsular or beyond), that follicular epithelial cell lesion is malignant.

Excluding papillary thyroid carcinoma, there is universal agreement that the diagnosis of follicular carcinoma requires the presence of capsular or vascular invasion of the tumor capsule or beyond the tumor capsule. Unfortunately, there is still controversy in regard to the interpretation of CI. While most authors require complete capsular transgression by tumor in order to diagnose capsular invasion [3, 4], other authorities will diagnose a given neoplasm as a carcinoma even in the presence of incomplete capsular transgression [5]. According to Chan [3], Fig. 1 depicts the various histologic appearances associated with the presence or absence of capsular invasion. According to this author [3], a given neoplasm should not be diagnosed as carcinoma if complete capsular penetration cannot be proven after extensive sampling except in one instance. This situation occurs when a satellite tumor nodule morphologically similar to the main tumor is lying just outside the tumor capsule (Fig. 1e). This appearance results from failure to identify the point of capsular penetration. It is noteworthy that not all authors agree that these satellite nodules represent CI [6]. In equivocal cases of $\mathrm{CI}$, the entire cancer irrespective of tumor size should be processed in the attempt to clarify whether CI is present. Deeper sections of the representative paraffin block(s) should be performed in the areas of concern in order to exclude CI [3].

\section{Angioinvasion or Lymph-Vascular Invasion}

The CAP guidelines for all cancer protocols calls for the use of the term lymph-vascular invasion (LVI) which is the terminology used in the Thyroid Cancer Protocol as well as all the protocols for the reporting of carcinomas of the entire upper aerodigestive tract, including the oral cavity, pharynx (oro-, naso- and hypopharynx), sinonasal tract, larynx and salivary glands.

While the criteria for capsular invasion are quite controversial, there is currently relatively good consensus on what constitutes lymph-vascular invasion. The majority of authors agree that lymph-vascular invasion should involve capsular or extra-capsular vessels (Fig. 2). These images (Fig. 2) depict intracapsular LVI with endothelialized thrombus, tumor thrombus with fibrin, and tumor thrombus attached to vessel wall. The tumor thrombus should protrude into the lumen and needs to be covered by endothelial cells (Fig. 2b). However, endothelialization is not a requirement if the tumor is attached to the vessel wall (Fig. 2c) or admixed with a fibrin thrombus (Fig. 2d). The point of attachment of the tumor to the vessel wall has to be identified for some authorities [7] to assure that free floating tumor 


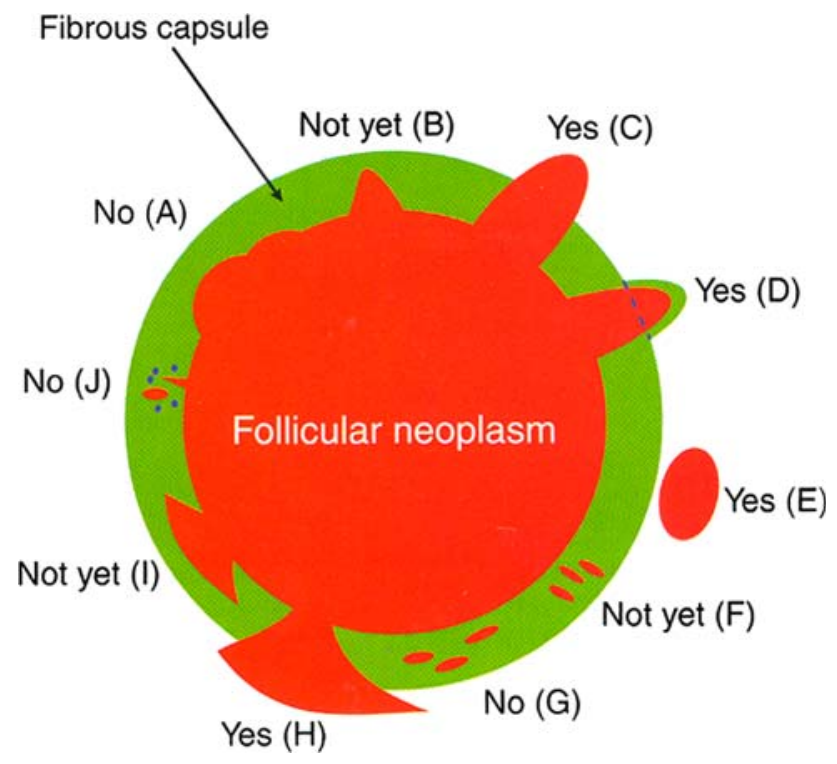

Fig. 1 Capsular invasion (CI): Schematic drawing for the interpretation of the presence or absence of CI. The diagram depicts a follicular neoplasm (orange) surrounded by a fibrous capsule (green). a bosselation on the inner aspect of the capsule does not represent CI; b sharp tumor bud invades into but not through the capsule suggesting invasion requiring deeper sections to exclude; c: tumor totally transgresses the capsule invading beyond the outer contour of the capsule qualifying as $\mathrm{CI}$; $\mathbf{d}$ : tumor clothed by thin (probably new) fibrous capsule but already extending beyond an imaginary (dotted) line drawn through the outer contour of the capsule qualifying as CI; e: satellite tumor nodule with similar features (architecture, cytomorphology) to the main tumor lying outside the capsule qualifying as $\mathrm{CI}$; f Follicles aligned perpendicular to the capsule suggesting invasion requiring deeper sections to exclude $\mathbf{g}$ follicles aligned parallel to the capsule do not represent $\mathrm{CI}$; $\mathbf{h}$ mushroom-shaped tumor with total transgression of the capsule qualifies as CI; i mushroom-shaped tumor within but not through the capsule suggests invasion requiring deeper sections to exclude; $\mathbf{j}$ neoplastic follicles in the fibrous capsule with a degenerated appearance accompanied by lymphocytes and siderophages does not represent CI but rather capsular rupture related to prior fine needle aspiration. Modified from: Chan JKC. Reprinted with permission [3]

artifactually displaced by the surgeon or the pathologist are not misinterpreted as LVI. Tumor in intra-tumoral or subcapsular vessels does not qualify for LVI and should not be interpreted as such (Fig. 2a).

\section{Extent of Capsular and Lymph-Vascular Invasion}

Follicular carcinomas of the thyroid gland, including its oncocytic variant (so-called Hürthle cell carcinoma), are subdivided into minimally and widely invasive carcinomas [4]. There is general agreement that follicular carcinomas with widespread gross invasion of the thyroid and peri-thyroid soft tissue should be labeled as widely invasive follicular carcinoma [3,4]. These tumors often lack complete encapsulation and have a poor prognosis [3]. In contrast, the criteria defining "minimally invasive" follicular carcinoma are controversial and still evolving. In some schemes, this designation refers to encapsulated lesions with capsular and/ or small caliber sized LVI even if the LVI is extensive [4]. The invasive foci in these limited invasive cancers are rarely, if ever, grossly identifiable. Defined as such minimally invasive follicular carcinomas have an overall good prognosis although some cases may recur and metastasize $[5,8]$. Identifying these "minimally invasive" follicular carcinomas may be crucial as some surgeons take a conservative management approach and treat these cancers by lobectomy alone followed by observation. Such an approach is held for all "minimally invasive" follicular carcinomas, including the oncocytic (Hürthle cell) category. However, the therapeutic approach for the diagnosis of any follicular carcinoma whether minimally invasive or not generally includes total thyroidectomy and post-operative radioactive iodine (RAI) therapy. It should be noted that the conservative approach of lobectomy and observation may risk undertreating those few minimally invasive tumors with a poor outcome. In order to detect those "minimally invasive" cases that recur, some authorities limit the use of the minimally invasive term to those carcinomas with capsular invasion only since their metastatic rate is close to $0[9,10]$. The designation "grossly encapsulated angioinvasive follicular carcinoma" has been suggested to encapsulated tumors with any foci of vascular invasion in view of their perceived higher risk of recurrence [11]. These authors feel that the presence of LVI, even in one or a few endothelial-lined vessels, negates a diagnosis of "minimal invasion" as these carcinomas once gaining access to any vessel have the capacity to behave in a more aggressive manner than those carcinomas with only capsular invasion without LVI [11]. Other authors base their definition of minimally invasive carcinoma on the number of foci of invasion especially vascular invasion. [10, 12-14]. In some studies, encapsulated follicular carcinoma, including the oncocytic variant, with four or more foci of vascular invasion have a significant recurrence rate $(47 \%$ for follicular oncocytic tumors) even if the foci of angioinvasion are microscopic [13, 14] (Fig. 3). These tumors are therefore called "grossly encapsulated follicular carcinoma with extensive angioinvasion". Another study showed that follicular oncocytic (Hürthle cell) carcinomas with a total of two foci of capsular/vascular invasion did not recur after a long follow up and should therefore be labeled as minimally invasive [12].

Irrespective of one's philosophy in regard to the definition of minimally invasive follicular carcinoma, the recommendation is that pathologists should report on the presence as well as the extent (focal, extensive) of capsular and lymph-vascular invasion. This approach has a dual advantage of collating the various terminologies suggested for these carcinomas, as well as and perhaps more importantly, providing a report that better assists the clinician in 


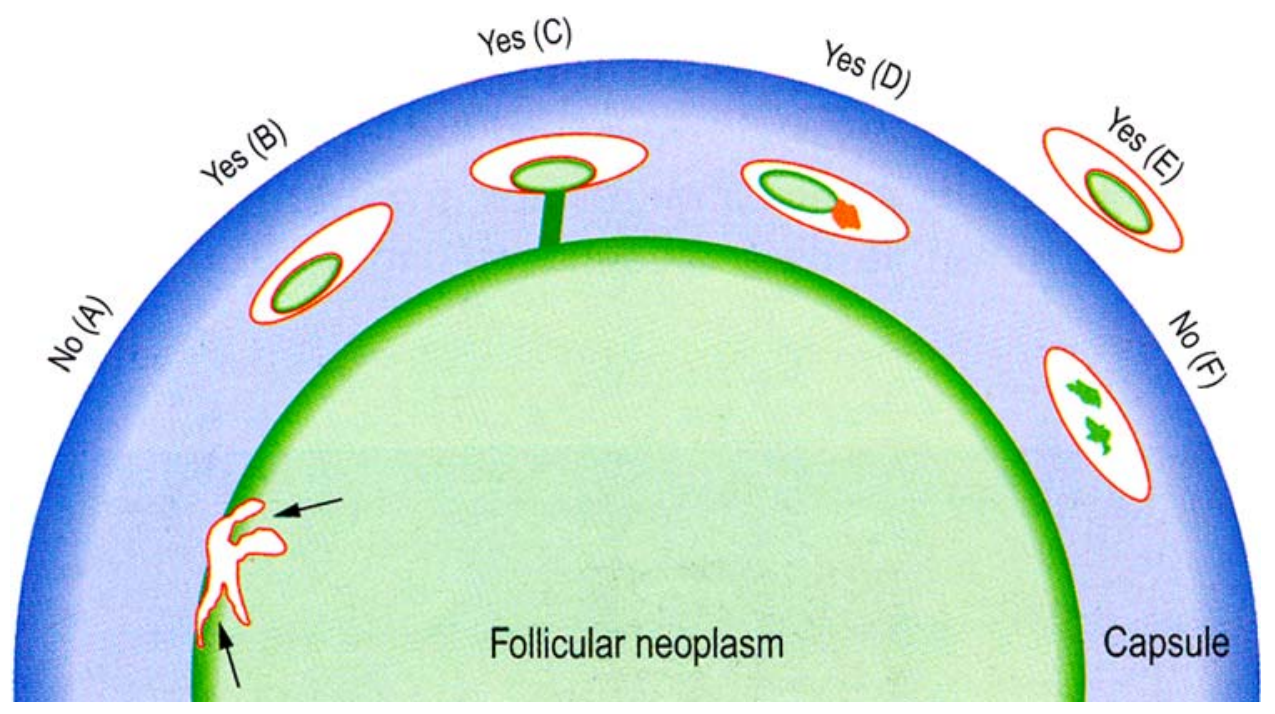

Fig. 2 Vascular invasion (VI): Schematic drawing for the interpretation of the presence or absence of VI. The diagram depicts a follicular neoplasm (green) surrounded by a fibrous capsule (blue). a Bulging of tumor into vessels within the tumor proper does not constitute VI. b Tumor thrombus covered by endothelial cells in intracapsular vessel qualifies as VI. c Tumor thrombus in intracapsular vessel considered as VI since it is attached to the vessel wall. d Although not endothelialized, this tumor thrombus qualifies for VI because it is accompanied by a fibrin thrombus. e Endothelialized tumor thrombus in vessel outside the tumor capsule represents VI. f Artefactual dislodgement of tumor manifesting as irregular tumor fragments into vascular lumen unaccompanied by endothelial covering or fibrin thrombus. Modified from: Chan JKC Reprinted with permission [3]

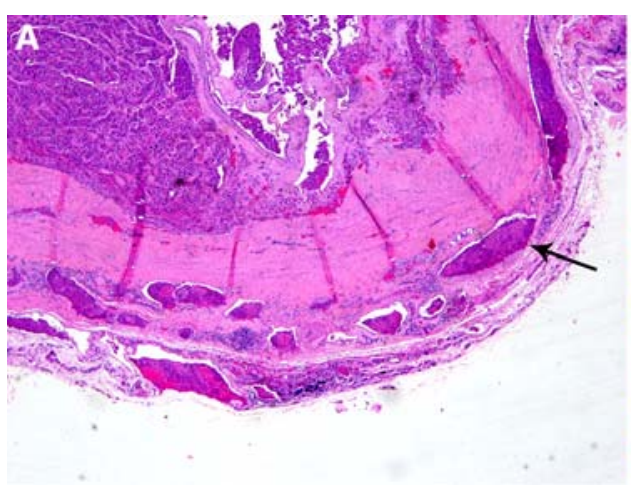

Fig. 3 Encapsulated follicular carcinoma (FC), oncocytic variant with multiple foci of microscopic vascular invasion (VI) and no gross invasion. In some classification schemes, these tumors are labeled as minimally invasive while others will use terms such as encapsulated angioinvasive $\mathrm{FC}$ or encapsulated $\mathrm{FC}$ with extensive angioinvasion to stress their potential for aggressive behavior. This 50 year old patient

assessing recurrence risk and, therefore, in deciding on the extent of surgical intervention (e.g., completion thyroidectomy) and the use of postoperative RAI therapy.

\section{Mitosis and Tumor Necrosis}

Increased mitotic activity and especially tumor necrosis are powerful indicators of adverse outcome in thyroid carcinomas of follicular origin. Asklen and Livolsi showed that tumor necrosis and mitotic rate $>2$ mitosis/10 high power fields indicate worse survival in papillary thyroid carcinoma

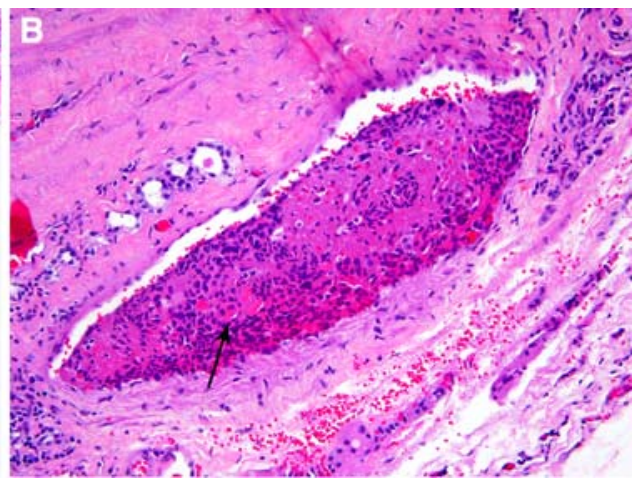

developed bone metastases 10 years after thyroidectomy. a Low power view showing multiple microscopic foci of VI in tumor capsule (arrow) and immediately outside the capsule. b high power view of a tumor thrombus (arrow) attached to vessel wall and covered by endothelial cells

( $P=0.028 ; P<0.00005$, respectively) [15]. Mitosis and tumor necrosis are also strongly associated with poorly differentiated thyroid carcinomas (PDTC). The latter type of carcinoma has a prognosis in between the indolent well differentiated papillary thyroid carcinomas and the almost universally lethal anaplastic carcinoma. Its definition is however subject to controversy. PDTC defined on the basis of high mitotic activity ( $\geq 5$ mitosis/10 high power fields, $400 \times$ ) and/or tumor necrosis have a disease specific survival of $60 \%$ at 5 years irrespective of the tumor architecture (Fig. 4) [16]. PDTC defined mainly on the 
basis of growth pattern alone (such as the tumors reported in the large Italian study by Volante et al.) also occupy an intermediate position at the prognostic level on the spectrum of thyroid carcinoma progression [17]. However, when Volante et al. developed a numeric scoring system whose most influential parameter was tumor necrosis, those neoplasms with necrosis had a much worse survival than those without [17]. Indeed, the overall survival curve of their most favorable subgroup even overlapped with that of patients with well-differentiated papillary and follicular carcinomas [17]. The overall survival of their most aggressive group (those patients whose neoplasms contained at least tumor necrosis) appears to be closer to that of PDTC defined by necrosis and/or a high mitotic rate [17]. Recently, a group of pathologists gathered in Turin, Italy in the attempt to provide a consensus view regarding PDTC [18].Their definition relied on the presence of solid growth but required the presence of at least one of the followings: convoluted nuclei, tumor necrosis and/or mitosis $\geq 3 / 10$ high power fields, $400 \times$. In this study as well, mitosis and tumor necrosis were very powerful indicator of poor outcome $(P=0.011 ; P<0.001$ respectively) while the type of PDTC (papillary vs non-papillary) was not. The value of mitosis and tumor necrosis is also emphasized by the fact that PDTC defined on the basis of mitoses and/or necrosis is the major cause of radioactive iodine (RAI) refractory, positron emission tomography (PET)-positive incurable thyroid carcinomas [19]. The importance of tumor necrosis in primary tumors is further validated by the fact that it was (along with extra-thyroid extension) the only independent variable associated with decreased overall survival within RAI refractory thyroid carcinomas [19].

From the above data, one can conclude that whatever definition is used for PDTC, it is very helpful to mention the presence of mitosis and tumor necrosis in the pathology report. It is however important to differentiate tumor necrosis from necrosis due to previous fine needle aspiration (FNA). Tumor necrosis has a "comedo-like" appearance composed of degenerating cytoplasm and punctuate, karyorectic nuclear debris (Fig. 4). In contrast, the presence of fibroblastic stromal reaction, evidence of hemorrhage or an identifiable needle tract in the necrotic area are attributable to reaction induced by prior FNA. Since the majority of thyroid cancers are well-differentiated lacking mitotic activity and necrosis, and the fact that PDTC is a rather uncommon diagnosis, the Thyroid Cancer Protocol recommends rather than requires the reporting of these data elements (i.e., mitotic activity and tumor necrosis).

\section{Extra-Thyroid Extension}

Extrathyroidal extension refers to involvement of the perithyroidal soft tissues by a primary thyroid cancer. On gross examination, the capsule may appear complete but evidence has shown that microscopically the capsule is focally incomplete in a majority of autopsy thyroid glands evaluated [20]. The capsule includes sizable vascular spaces as well as small peripheral nerves and is continuous with the pretracheal fascia. [21]. In practice, since the fibrous capsule of the thyroid is often incomplete, the criteria for
Fig. 4 Tumor necrosis in thyroid carcinoma with various growth patterns. a Follicular growth pattern with tumor necrosis $(\mathrm{N})$. b Papillary architecture with necrosis $(\mathrm{N})$. c Tumor with a predominantly solid growth pattern and a large area of necrosis $(\mathrm{N})$. d Tumor with an insular pattern. Necrosis was present elsewhere in the specimen. Reproduced with permission from Hiltzik et al. [14]
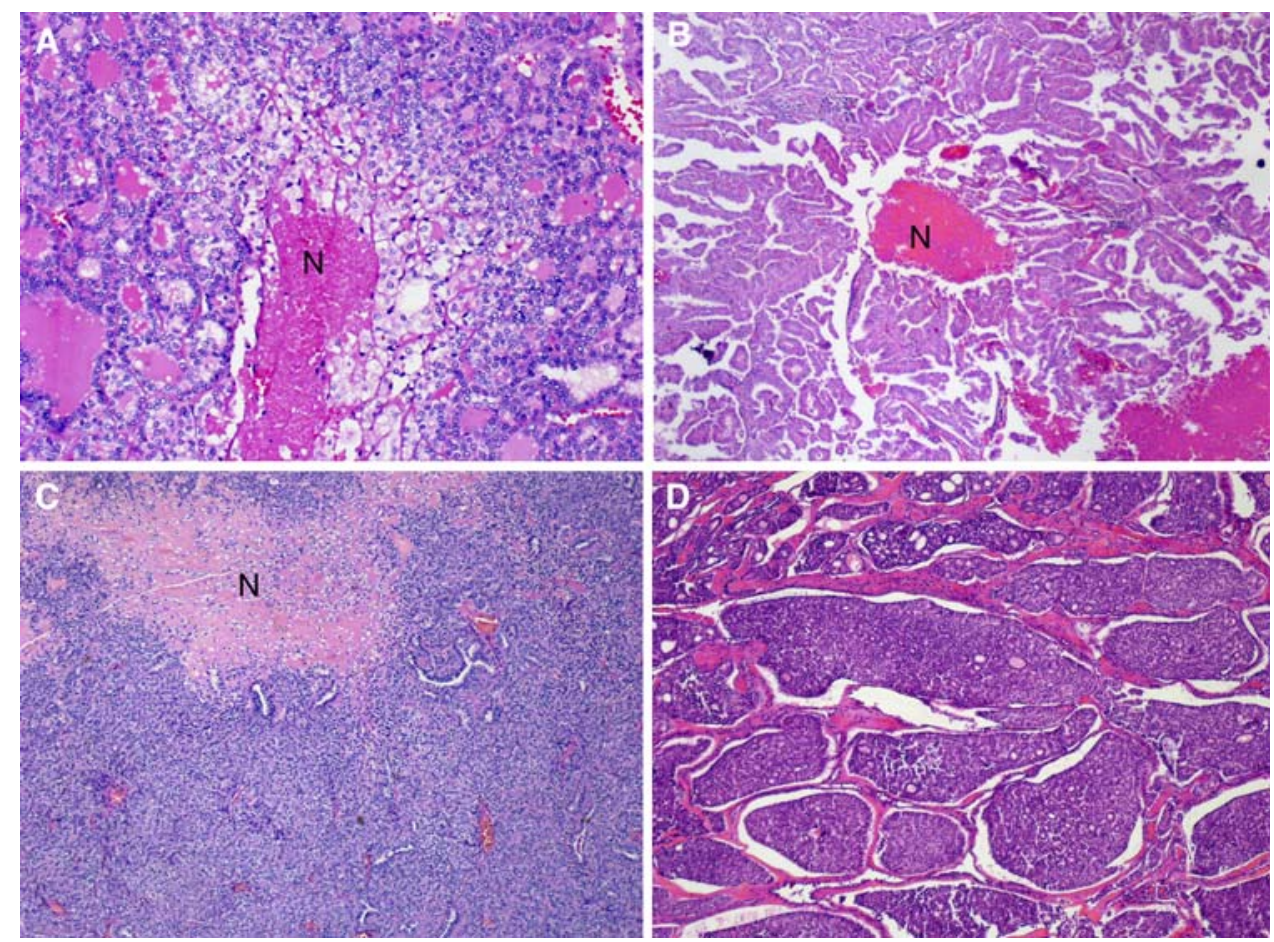


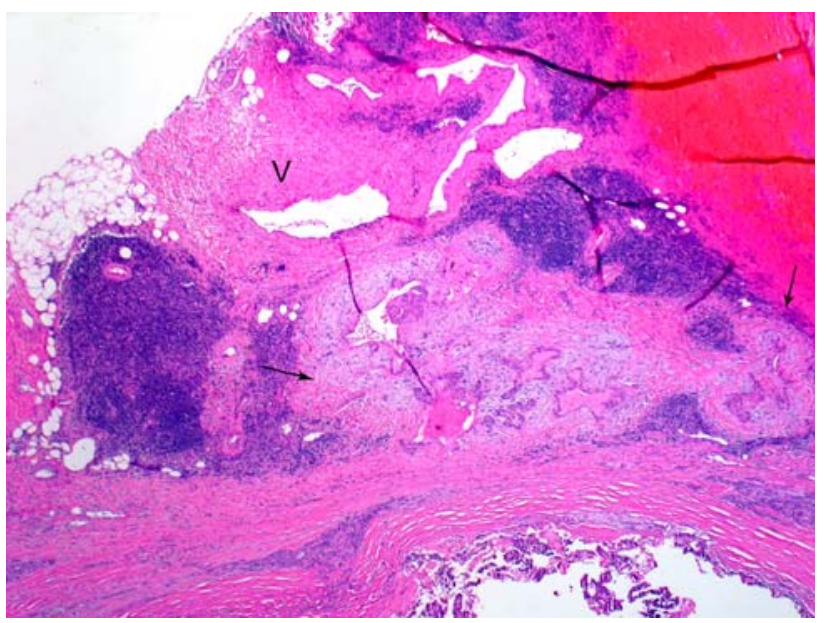

Fig. 5 Minimal extra-thyroid extension (ETE) into peri-thyroid fat. The focus of ETE (between arrows) has a desmoplastic reaction (pale staining fibrous tissue). Note nearby thick walled blood vessel (V). Both findings are indicative of ETE

defining (minimal) extrathyroidal extension may be problematic and subjective. Diagnostic findings for minimal extrathyroidal extension includes the presence of carcinoma extending into perithyroidal soft tissues, including infiltration of adipose tissue and skeletal muscle, as well as around (and into) sizable vascular structures and nerves. Extension into adipose tissue can be problematic given the fact that adipose tissue can be found within the thyroid gland proper under normal conditions and also may be a component of a variety of thyroid lesions including carcinomas. [22]. As such, the presence of adipose tissue in association with a thyroid carcinoma should not be mistaken for extrathyroidal extension. Some authorities only accept invasion of skeletal muscle as the identifier for extrathyroidal extension. However, similar to adipose tissue in the thyroid, the presence of skeletal muscle may be seen in the thyroid gland under normal conditions, especially in relation to the isthmus portion of the thyroid gland, as well as in a variety of pathologic conditions [22]. If present, a desmoplastic response may be a helpful finding in the determination of extrathyroidal extension (Fig. 5). The identification of thick-walled vascular spaces and/or small peripheral nerves in association with adipose tissue may be of greater assistance as these structures are not located in the thyroid gland proper and their presence would be helpful in determining whether the carcinoma is extrathyroidal in extent (Fig. 5).

While minimal extra-thyroid extension can be difficult to identify, extensive extra-thyroid extension is always obvious and easily diagnosed by the surgeon during the thyroidectomy. Extensive extrathyroid extension is defined by the presence of carcinoma well beyond the thyroid gland proper with direct invasion (i.e., not metastasis) into one or more of the following structures:subcutaneous soft tissues; adjacent viscera, including the larynx, trachea and/ or esophagus; the recurrent laryngeal nerve, carotid artery or mediastinal blood vessels. Many studies have shown that carcinomas with extensive extra-thyroid extension have a much worse survival than those with minimal extra-thyroid extension [3, 23]. Moreover, some studies have found a similar outcome in patients with minimal versus no extrathyroid extension [23].

Based on the above data, it is therefore mandatory to report on the extent (minimal versus extensive) of extrathyroid extension.

\section{Resection Margins}

Few published studies have addressed the influence of margin status and patient outcome. Most surgeons, endocrinologists, and nuclear medicine specialists require knowledge of positive margins, i.e., tumor extending to surgical resection edge. While this makes intuitive sense and it is recommended that a positive margin be mentioned in the final pathology report, meticulous studies on the effect of positive margins and outcome in large series of patients with long-term follow-up are lacking.

At the present time, there is no need to report the distance of tumor to closest resection margin. Indeed, there is no data to date on the prognostic value of close margins as an independent or co-variable.

\section{Lymph Node Metastases}

Although controversy still exists in regard to the prognostic value of nodal metastases in papillary thyroid carcinomas, the reporting on lymph node status is mandatory since positive nodal metastases most often lead to RAI therapy. The pathologist should also comment on the presence or absence of extranodal extension since the latter was shown to increase the risk for distant metastases and death [24].

\section{Papillary Thyroid Microcarcinoma}

This variant of papillary thyroid carcinoma is defined as any focus measuring $\leq 1 \mathrm{~cm}$. [4] Such papillary thyroid microcarcinomas usually are incidentally identified in thyroid glands removed for other reasons. There is general agreement that no additional therapy is needed for these incidentally identified foci of thyroid papillary microcarcinoma and, in order to avoid overtreatment, it is worthwhile to consider indicating in the pathology report that these foci have an extremely favorable prognosis and should not be used as a reason for additional therapy (e.g., completion thyroidectomy and RAI). Given their rather common identification in all thyroid gland resections and their indolent biologic behavior, it is not the recommendation of 
the CAP Thyroid Cancer Protocol to issue a protocol for each case in which incidental papillary thyroid microcarcinomas are found. An exception to such practice would be considered in those examples of papillary thyroid carcinomas measuring $\leq 1 \mathrm{~cm}$ but representing the primary reason a lobe/gland was removed. The tumor could have been discovered clinically (palpable, visible nodule) or by imaging. Given the more sophisticated diagnostic (e.g., imaging) modalities currently available, smaller (i.e., $<1 \mathrm{~cm}$ ) lesions are being identified and resected. In such circumstances, where the primary reason for thyroid surgery is to excise a subcentimeter focus of PTC, then reporting should follow the CAP Thyroid Protocol.

Although usually extremely indolent, papillary thyroid microcarcinomas may exceptionally behave aggressively with spread to lymph node or distant sites [25]. Such aggressive papillary thyroid microcarcinomas usually harbor their metastases at presentation [25]. The presence of two or more foci of papillary thyroid microcarcinonas, and aggressive features related to the primary tumor such as lymph-vascular invasion, extra-thyroid extension and "aggressive" morphology (e.g., tall cell features) may trigger full blown treatment including total thyroidectomy and RAI therapy. Such management does not appear to be justified at this time as there is insufficient data in the literature (long term follow-up) on these papillary thyroid microcarcinomas with "aggressive" features in the primary to justify such a therapeutic approach. It is our recommendation that the designation of papillary thyroid microcarcinoma should not be applied to children and adolescents under 19 years old as a significant number of these subcentimeter papillary carcinomas occurring in the pediatric population display extrathyroidal extension and distant metastases [26].

\section{Conclusion}

The updated CAP protocol is a step toward improving the clinical value of the pathologic reporting of thyroid carcinomas. An accurate assessment of the extent of invasion of the tumor capsule, especially lymph-vascular invasion, is an important element in the reporting of thyroid carcinomas. Meticulous microscopic examination of TC is no longer an academic exercise but a necessity in the management of these malignancies. Proliferative assessment of the tumor (i.e., mitosis and necrosis) is of high prognostic value in the determination of a poorly-differentiated thyroid carcinoma. The extremely indolent behavior of papillary thyroid microcarcinoma should be communicated to the clinician in order to avoid overtreatment.

There are still unresolved issues in the histopathologic diagnosis of thyroid carcinomas. Large clinico-pathologic studies with long term follow up are still needed in order to increase the impact of histopathology on the prognosis and management of TC. With the advent of molecular diagnostics the anticipation is that many of these controversial issues will be resolved but until that time, pathologists must rely on morphology in the assessment and reporting of thyroid carcinomas.

\section{References}

1. Lloyd RV, Erickson LA, Casey MB, et al. Observer variation in the diagnosis of follicular variant of papillary thyroid carcinoma. Am J Surg Pathol. 2004;28:1336-40.

2. Elsheikh TM, Asa SL, Chan JK, et al. Interobserver and intraobserver variation among experts in the diagnosis of thyroid follicular lesions with borderline nuclear features of papillary carcinoma. Am J Clin Pathol. 2008;130:736-44.

3. Chan JKC. Tumors of the thyroid and parathyroid glands. In: Fletcher CDM, editor. Diagnostic Histopathology of Tumors. 3rd ed. London, England: Churchill Livingston; 2007. p. 997-1078.

4. Rosai J, Carcangiu ML, Delellis RA. Tumors of the Thyroid Gland, Atlas of Tumor Pathology. Washington DC: Armed Forces Institute of Pathology; 1992.

5. Thompson LD, Wieneke JA, Paal E, et al. A clinicopathologic study of minimally invasive follicular carcinoma of the thyroid gland with a review of the English literature. Cancer. 2001;91:505-24.

6. Suster S. Thyroid tumors with a follicular growth pattern: problems in differential diagnosis. Arch Path lab med. 2006;130:984-8.

7. Khan A, Nose V. Pathology of the thyroid gland. In: Lloyd RV, editor. Endocrine Pathology. Differential diagnoses and molecular advances. Totowa, NJ: Humana Press; 2003. p. 175.

8. Evans HL, Vassilopoulou-Sellin R. Follicular and Hurthle cell carcinomas of the thyroid: a comparative study. Am J Surg Pathol. 1998;22:1512-20.

9. Van Heerden JA, Hay ID, GOellner JR, et al. Follicular thyroid carcinoma with capsular invasion alone: a non-threatening malignancy. Surgery. 1992;112:1130-6.

10. Lang W, Choritz H, Hundeshagen H. Risk factors in follicular thyroid carcinomas. A retrospective follow-up study covering a 14-year period with emphasis on morphological findings. Am J Surg Pathol. 1986;10:246-55.

11. Baloch ZW, Livolsi VA. Follicular-patterned lesions of the thyroid: the bane of the pathologist. Am J Clin Pathol. 2002;117: $143-50$.

12. Stojadinovic A, Ghossein RA, Hoos A, et al. Hurthle cell carcinoma: a critical histopathologic appraisal. J Clin Oncol. 2001;19: 2616-25.

13. Ghossein RA, Hiltzik DH, Carlson DL, et al. Prognostic factors of recurrence in encapsulated Hurthle cell carcinoma of the thyroid gland: a clinicopathologic study of 50 cases. Cancer. 2006; 106:1669-76.

14. Collini P, Sampietro G, Pilotti S. Extensive vascular invasion is a marker of risk of relapse in encapsulated non-Hurthle cell follicular carcinoma of the thyroid gland: a clinicopathological study of 18 consecutive cases from a single institution with a 11year median follow-up. Histopathology. 2004;44:35-9.

15. Asklen LA, LiVolsi VA. Prognostic significance of histologic grading compared with subclassification of papillary thyroid carcinoma. Cancer. 2000;88:1902-8.

16. Hiltzik D, Carlson DL, Tuttle RM, et al. Poorly differentiated thyroid carcinomas defined on the basis of mitosis and necrosis: a clinicopathologic study of 58 patients. Cancer. 2006;106: 1286-95. 
17. Volante M, Landolfi S, Chiusa L, et al. Poorly differentiated carcinomas of the thyroid with trabecular, insular, and solid patterns: a clinicopathologic study of 183 patients. Cancer. 2004;100:950-7.

18. Volante M, Collini P, Nikiforov YE, et al. Poorly differentiated thyroid carcinoma: the Turin proposal for the use of uniform diagnostic criteria and an algorithmic diagnostic approach. Am J Surg Pathol. 2007;31:1256-64.

19. Rivera M, Ghossein RA, Schoder H, et al. Histopathologic characterization of radioactive iodine-refractory fluorodeoxyglucose-positron emission tomography-positive thyroid carcinoma. Cancer. 2008;113:48-56.

20. Komorowski RA, Hanson GA. Occult thyroid pathology in the young adult: an autopsy study of 138 patients without clinical thyroid disease. Hum Pathol. 1988;19:689-96.

21. Standring S. Thyroid gland. In: Standring S, editor. Gray's anatomy. The anatomical basis of clinical practice. 39th ed. Edinburgh: Elsevier Churchill Livingstone; 2005. p. 560-4.
22. Carcangiu ML. Thyroid. In: Mills SE, editor. Histology for pathologists. 3rd ed. Philadelphia: Lippincott Williams \& Wilkins; 2007. p. 1129-48.

23. Ito $\mathrm{Y}$, Tomoda $\mathrm{C}$, Uruno $\mathrm{T}$, et al. Prognostic significance of extrathyroid extension of papillary thyroid carcinoma: massive but not minimal extension affects the relapse-free survival. World $\mathrm{J}$ Surg. 2006;30:780-6.

24. Yamashita H, Noguchi S, Murakami N, et al. Extracapsular invasion of lymph node metastasis is an indicator of distant metastasis and poor prognosis in patients with thyroid papillary carcinoma. Cancer. 1997;80:2268-72.

25. Lo CY, Chan WF, Lang BH, et al. Papillary microcarcinoma: is there any difference between clinically overt and occult tumors? World J Surg. 2006;30:759-66.

26. Rosai J, LiVolsi VA, Sobrinho-Simoes M, et al. Renaming papillary microcarcinoma of the thyroid gland: the porto proposal. Int J Surg Pathol. 2003;11:249-51. 